\title{
A Compact Tunable Bandpass Filter Using Coupled Metamaterial Resonators with Varactor Diode
}

\author{
Gi-rae Kim, Member, KIMICS
}

\begin{abstract}
In this paper, we present a novel tunable microstrip bandpass filter based on split ring resonators (SRRs). The varactors are reverse-biased semiconductor diode, and are connected between the concentric rings of the SRR. An individual varactor loaded SRR based bandpass tunable filter module is analyzed. Then a second order tunable filter with $7 \%$ fractional bandwidth and a tuning range from 2.75 to $2.86 \mathrm{GHz}$ is assembled from basic filter modules. The simulator HFSS (V10) is used to design the tunable filter and to simulate. The results show good characteristics is created.
\end{abstract}

Index Terms - Tunable filter, Bandpass filter, Split ring resonator (SRR), Varactor diode.

\section{INTRODUCTION}

FREQUENCY tunable filters are of great interest in the design of multifunctional wireless and satellite communication systems. These tunable components provide compactness and cost reduction to the RF frontends since they combine multiple bands in one unit to save space and material. There have been techniques reported such as radio frequency micro-electromechanical system switches (RF MEMS) [1,2], ferromagnetic materials [3], or semiconductor varactors [4-6] to design tunable filters. Among those techniques, ferromagnetic material and RF MEMS based tunable filters are expensive and the fabrication process can be challenging. Semiconductor varactor loaded microstrip tunable filters, on the other hand, are compact, low-cost and easy to integrate. All these advantages make varactor loaded filters very attractive to be used in multiband wireless front-ends. To further miniaturize the filter design, we choose to use planar split ring resonators (SRRs). SRRs are known for their compactness since they resonate at a dimension much smaller than a wavelength [7]. They are high-Q resonators and can be excited by a time-varying electric or magnetic field.

In 1968, Veselago first investigated theoretically materials with simultaneously negative permittivity and permeability, or left-handed (LH) materials. Recently,

\footnotetext{
Manuscript received July 9, 2010; revised July 26, 2010; accepted July 31, 2010.

Gi-rae Kim is with the Department of Electronics Engineering, Silla University, Busan, 617-736, Korea, Email: grkim@silla.ac.kr)
}

these materials have known a tremendous renew of interest, since the demonstration of their practical in composites including negative-wires and negative-ring resonators. Although LH effects have also been demonstrated in some parts of the band structure of photonic crystals, LH materials generally have diffraction sites with inter-distances much smaller than wavelength, so that they can be effectively considered as homogeneous media.

The unique properties of LH metamaterials, verified by full-wave analysis, are promising for a diversity of optical/microwave applications, such as new types of beam steerers, modulators, band-pass filters, superlenses, microwave components and antennas. However, the LH structures presented originally were impractical for microwave applications, because of their too lossy and too narrow bandwidth characteristics, and alternative theories are desirable to gain a deeper insight into their behavior. Itoh present here a transmission line (TL) approach of LH materials based on nonresonant components, describe a procedure to realize an artificial LH-TL with low losses and broad bandwidth and demonstrate a microstrip implementation of this line using interdigital capacitors and stub inductors.

A tunable bandpass tunable filter based on varactor loaded SRRs (VLSRRs) is presented in [9] with a fairly high insertion loss. The basic tunable bandpass filter module that we use to design the second order filter in this work is similar to VLSRR, except in our module the separation between the split rings of the SRR is uniform. The purpose of this study is to present a low loss tunable bandpass filter using varactor loaded SRRs.

\section{METAMATERIAL RESONATORS}

The basic cell of the structure is represented in Fig. 1. R-SRRs can be modeled as LC resonant tanks that can be externally driven by a magnetic field and are therefore able to inhibit signal propagation in a certain narrow band if they are properly oriented. All dimensions of structure Fig. $1(\mathrm{c})$ are $\mathrm{B}=50 \mathrm{~mm}, \mathrm{~s}=0.2 \mathrm{~mm}$, $\mathrm{D}=16 \mathrm{~mm}, \quad \mathrm{w}=2.36 \mathrm{~mm}, \quad$ and $\mathrm{g}=\mathrm{c}=\mathrm{d}=1.5 \mathrm{~mm}$. The electrical characteristic of R-SRRs is represented in Fig. 2(a). The R-SRR resonator equivalent circuit model is depicted in Fig. 2(b). 


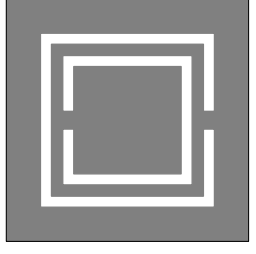

(a)

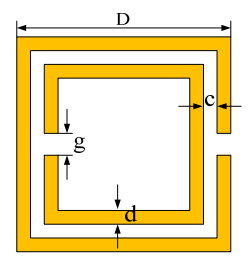

(c)

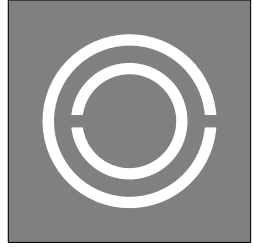

(b)

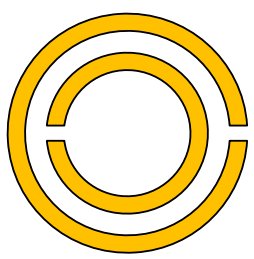

(d)
Fig.1 R-SRR element (a)(b) Complementary Split Ring Resonator Cell (CSRR Cell) (c)(d) Split Ring Resonator Cell (SRR Cell)

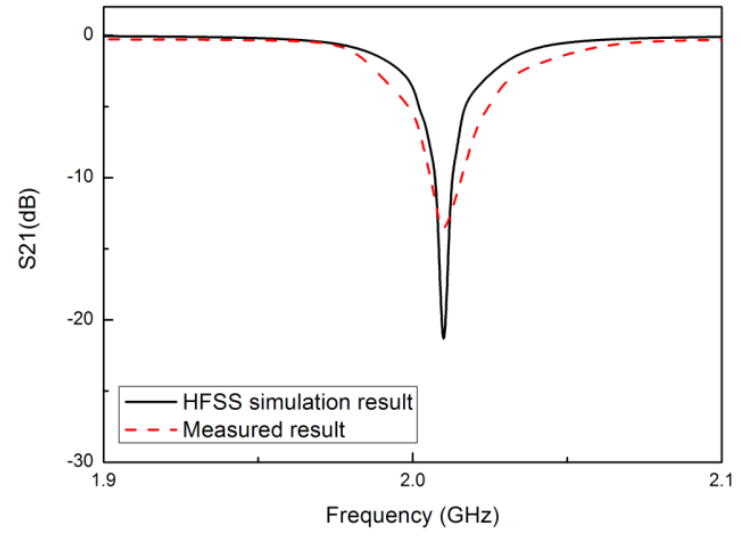

(a)

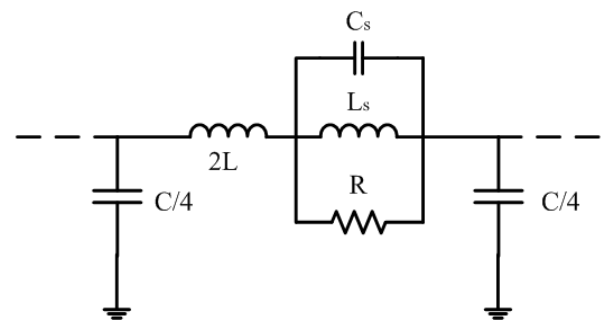

(b)

Fig. 2 Characteristics (a) and Equivalent model (b) of RSRR cell

The insertion loss of a conventional bandstop resonator is given by:

$$
I L=10 \log \left[1+\frac{1+4 R}{4 R^{2}+4 Q_{u}^{2} R^{2}\left[\frac{\omega}{\omega_{0}}-\frac{\omega_{0}}{\omega}\right]}\right] \mathrm{dB}
$$

The 3-dB bandwidth is:

$$
\Delta_{3 \mathrm{~dB}}=\omega_{0} \sqrt{\frac{1+4 R-4 R^{2}}{4 Q_{u}^{2} R^{2}}}
$$

Now let $\frac{\omega_{0}}{\Delta_{3 d B}}=\frac{Q_{u}}{2}$ equal to (2)

$$
\frac{2}{Q_{u}}=\sqrt{\frac{1+4 R-4 R^{2}}{4 Q_{u}^{2} R^{2}}}
$$

Therefore at $\omega_{0}$ :

$$
I L=10 \log \left[1+\frac{1+4 R}{4 R^{2}}\right]
$$

\section{TUNABLE BANDPASS FILTER DESIGN}

A tunable filter block module with resonant frequency located in the vicinity of $2.8 \mathrm{GHz}$ is designed. The basic tunable block is shown in Fig. 3. All dimensions of Fig. 3 structure are: $\mathrm{w}_{1}=1.5 \mathrm{~mm}, \mathrm{w}_{2}=10 \mathrm{~mm}, \mathrm{w}_{3}=1.5 \mathrm{~mm}$, $\mathrm{D}=12 \mathrm{~mm}, \mathrm{~g}=1 \mathrm{~mm}, \mathrm{c}=\mathrm{d}=1.5 \mathrm{~mm}$. The spacing between concentric rings of SRR is set to $1.5 \mathrm{~mm}$ to leave enough space for a tuning diode. The width of the gap $\mathrm{w}_{1}$ is set narrow enough to get an insertion loss less than $2 \mathrm{~dB}$ at the passband. Teflon high frequency laminate (relative permittivity $=2.17$, thickness $=0.7874 \mathrm{~mm}$ ) is used as the substrate. The characteristic impedance of the input line is set to $50 \mathrm{Ohm}$. The commercially available tuning diode Skyworks SMV1233 is chosen as the varactor. The varactor diode junction capacitance can be tuned between $0 \mathrm{pF}$ and $5.08 \mathrm{pF}$ by changing its biasing voltage. The excitation and coupling between the SRRs are through the magnetic field. The tuning diodes are placed between two concentric split rings of each SRR so that it has minimum effect on the excitation and coupling between the SRRs. The simulation results (performed with HFSS V10) for the scattering parameters of the basic tunable bandpass filter module for various varactor capacitance values are illustrated in Fig. 4. In the simulation, an ideal varactor that doesn't permit current to leak into the inner ring of the SRR is placed between concentric rings of the SRR. When the varactor capacitance is varied from $0 \mathrm{pF}$ to $4 \mathrm{pF}$, the resonant frequency is tuned from $2.86 \mathrm{GHz}$ to 2.73 GHz. The simulated insertion loss is low and constant through the tuning range. 
The basic filter model equivalent circuit is depicted in Fig. 5. $\mathrm{L}_{\mathrm{s}}$ is effective inductance of $\mathrm{SRR}$ cell; $\mathrm{C}_{\mathrm{s}}$ is effective capacitance of SRR cell. $\mathrm{C}_{\mathrm{v}}$ is the equivalent model for two varactor diodes. The parameters affecting the characteristic of R-SRR cell include the shape, size, gap and so on. $\mathrm{C}_{\mathrm{g} 1}$ and $\mathrm{C}_{\mathrm{g} 2}$ are effective gap capacitances between the arch and the SRR.

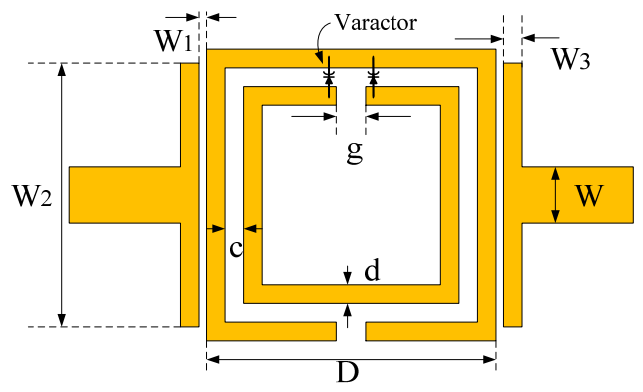

Fig. 3 Schematic of basic filter model

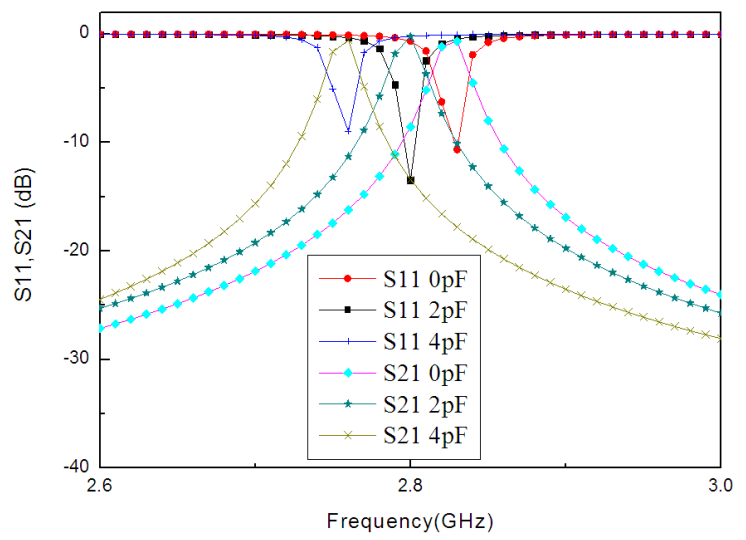

Fig.4 Characteristics results of basic filter model

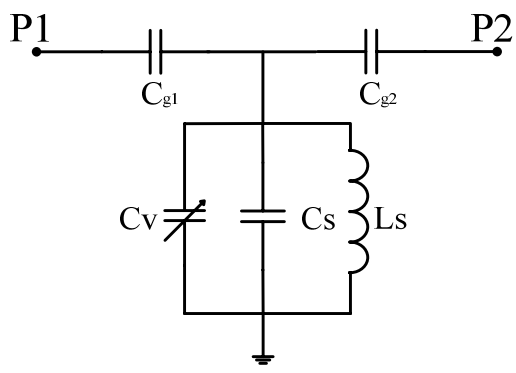

Fig. 5 Equivalent model of basic filter model

The filter synthesis using coupled resonators approach presented in Hong and Lancaster [10] is applied to design the tunable filter. We used the generic coupled resonators scheme of second order bandpass filter structure with transmission zeroes located at the upper side of the passband. The design is performed by calculating the coupled resonator based bandpass filter parameters, i.e. coupling coefficients and external quality factor presented in Hong and Lancaster [10]. A second order tunable bandpass filter using varactor loaded SRRs with a passband is proposed in Fig. 6 .

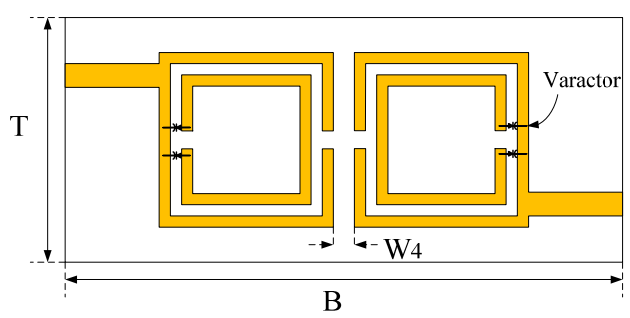

Fig. 6 Schematic of second order filter model

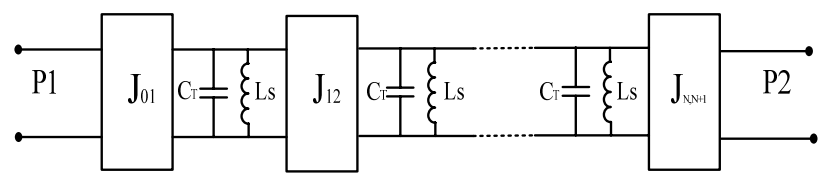

Fig. 7 Equivalent circuit of bandpass filter

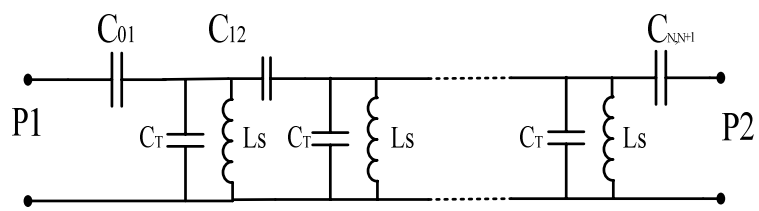

Fig. 8 Equivalent circuit of bandpass filter with circuit implementation

Admittance $(J)$ inverters are useful for bandpass or bandstop filters with narrow $(<10 \%)$ bandwidths. A J inverter can be constructed using a quarter-wave transformer of the appropriate characteristic impedance. Fig. 7 shows the equivalent circuit of general bandpass filter circuit using shunt resonators with admittance inverters. The function of admittance inverters is to convert alternate shunt resonators to series resonators; the extra inverters at the ends serve to scale the impedance level of the filter to a realistic level. Fig. 8 shows the equivalent circuit with replacement of admittance inverters.

Using an analysis of the equivalent circuit, the admittance inverter can be derived as:

$$
\begin{aligned}
& Z_{0} J_{01}=\sqrt{\frac{\pi \Delta}{4 g_{1}}} \\
& Z_{0} J_{n, n+1}=\frac{\pi \Delta}{4 \sqrt{g_{n} g_{n+1}}}
\end{aligned}
$$




$$
Z_{0} J_{N, N+1}=\sqrt{\frac{\pi \Delta}{4 g_{N} g_{N+1}}}
$$

Similarly, the coupling capacitor values can be found as

$$
\begin{aligned}
& C_{01}=\frac{J_{01}}{\omega_{0} \sqrt{1-\left(Z_{0} J_{01}\right)^{2}}} \\
& C_{n, n+1}=\frac{J_{n, n+1}}{\omega_{0}} \\
& C_{N, N+1}=\frac{J_{N, N+1}}{\omega_{0} \sqrt{1-\left(Z_{0} J_{N, N+1}\right)^{2}}}
\end{aligned}
$$

The resonant part of bandpass filter in practical is often used in microstrip circuits consists of an opencircuit $\lambda / 2$ transmission line. The capacitance of the quivalent circuit is

$$
C_{S}=\frac{\pi}{2 \omega_{0} Z_{0}}
$$

The inductance of the equivalent circuit is

$$
L_{S}=\frac{1}{\omega_{0}^{2} C}
$$

where $\omega_{0}=2 \pi f_{0} . f_{0}$ is the center frequency of bandpass filter. $Z_{0}$ is the impedance of bandpass filter, $50 \mathrm{Ohm}$.

The second order tunable bandpass filter passband centered at $2.8 \mathrm{GHz}$ is designed. The layout and the dimensions of the filter are shown in Fig. 6. The dimensions: $\mathrm{T}=24 \mathrm{~mm}, \mathrm{~B}=35 \mathrm{~mm}$, and $\mathrm{w}_{4}=1 \mathrm{~mm}$. The other values are the same with before section.

The simulated S-parameter results of the third order tunable bandpass filter are depicted in Fig. 9, respectively. The fabricated filter can be tuned from 2.75 to $2.86 \mathrm{GHz}$ by changing the biasing voltage from $0 \mathrm{~V}$ to $15 \mathrm{~V}$. The simulated insertion loss of $\mathrm{S} 21$ at $2.75 \mathrm{GHz}$ is $2.65 \mathrm{~dB}$ and $1.98 \mathrm{~dB}$ at $2.86 \mathrm{GHz}$. For the applications where such difference is acceptable, the tuning range and bandwidth of the filter are calculated to be 10.9 and $7 \%$, respectively, using the simulated S21 data of the fabricated filter shown in Fig. 9. In Fig. 9, with capacitance higher, the resonator frequency is lower.

Fig. 10 shows the second order filter model equivalent circuit. $L_{s}$ is effective inductances of $S R R$ cell; $C_{s}$ is effective capacitances of SRR cell. $\mathrm{C}_{\mathrm{v}}$ is the equivalent model for two varactor diodes. $\mathrm{C}_{01}$ and $\mathrm{C}_{23}$ are the effective gap capacitances between the arch and the SRR. $\mathrm{C}_{12}$ is the coupling capacitance for two cells. Despite the high insertion loss in the lower frequency band, the tuning range of the varactor loaded SRR filter is fairly good and is five times of the filter bandwidth.

We have mentioned the bandpass theory with $J$ inverter implemented. So in Fig. 11, equivalent capacitors $\mathrm{C}_{01}, \mathrm{C}_{12}$, $\mathrm{C}_{23}$ can be replaced by $J$ inverters $J_{01}, J_{12}, J_{23}$. According the implement of equations (5)-(13), we can get $\mathrm{C}_{01}, \mathrm{C}_{12}$, $\mathrm{C}_{23}$ values. At the same time, $\mathrm{C}_{\mathrm{s}}$ and $\mathrm{L}_{\mathrm{s}}$ can be calculated too. All of the exact values are shown in Fig. 12. As values are derived, with ADS (Advanced Design System), we can get the simulation result with lumped circuit elements as shown in Fig. 13. From Fig. 13, the ADS simulation result and HFSS simulation result are compared. The centre frequency is exactly the same. The bandwidth of filter has a little different. The reason of this is different simulation form get a little different characteristic.

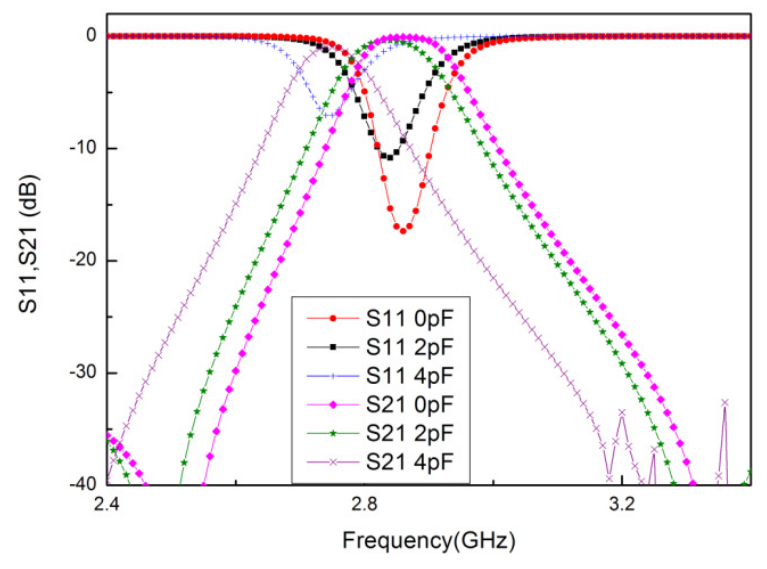

Fig. 9 Characteristics results of second order filter model

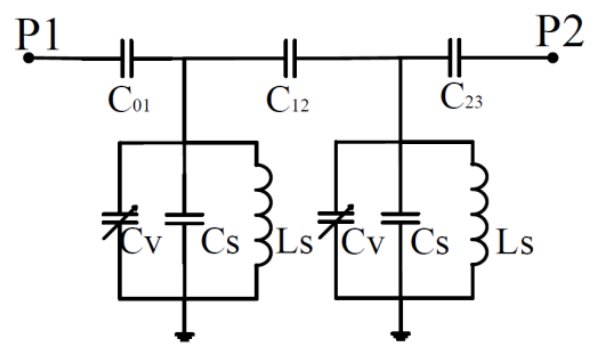

Fig. 10 Equivalent model of second order filter model 


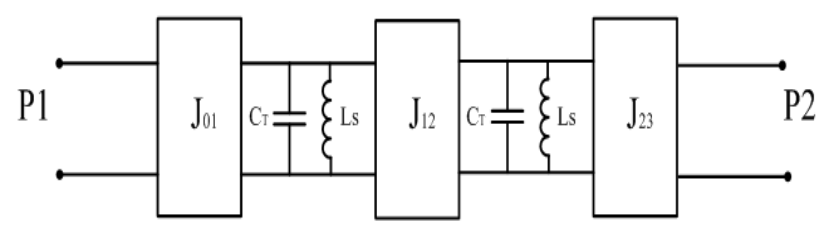

Fig. 11 Equivalent Model of second order filter model

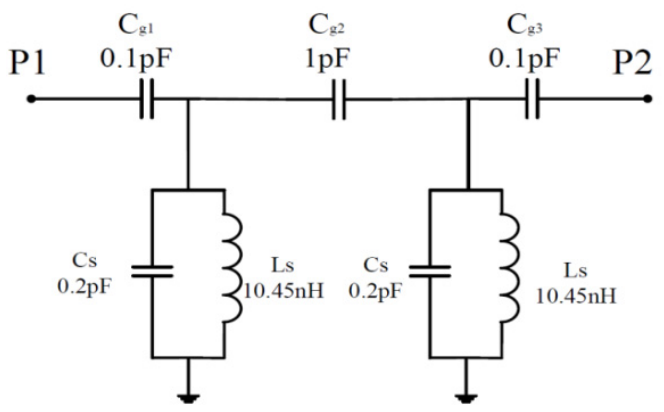

Fig. 12 The lumped element value for equivalent circuit

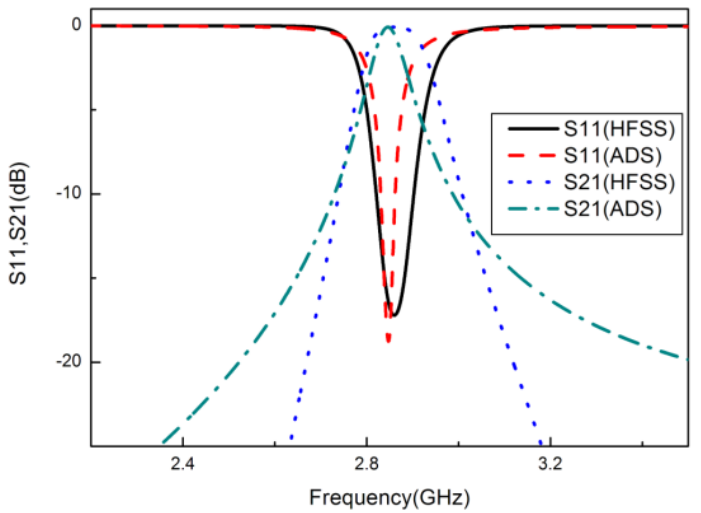

Fig. 13 Simulation Result without varactor

\section{CONCLUSIONS}

A compact planar tunable microstrip bandpass filter using varactor loaded SRRs has been implemented. A reverse-biased diode with voltage dependent variable nonlinear capacitance is used as the varactor. A prototype second order tunable bandpass filter using two coupled resonator with a passband located at around $2.8 \mathrm{GHz}$ is designed, fabricated and tested. The dimension of the second order fabricated tunable filter is $24 \mathrm{~mm}$ by $35 \mathrm{~mm}$, and is less than $1 / 3$ of the resonant wavelength. Therefore the miniaturization factor is about 3.5 compared to a third order half-wavelength coupled line filter.

\section{REFERENCES}

[1] C. T. -C. Nguyen, L. P. B. Katehi, and G. M. Rebeiz, "Micromachined devices for wireless communications", Proc. of the IEEE, vol. 86, pp. 1756-1768, 1998.

[2] K. Entesari and G.M. Rebeiz, "A differential 4-bit 6.5-10-GHz RF MEMS tunable filter", IEEE Trans Microwave Theory Tech., vol. 53, pp. 1103-1110, 2005.

[3] Y. Ishikawa, T. Nishikawa, T. Okada, S. Shinmura, Y. Kamado, F. Kanaya, and K. Wakino, "Mechanically tunable MSW bandpass filter with combined magnetic units", IEEE MTT-S Int. Microwave Symp. Digest, pp. 143-146, 1990.

[4] A. R. Brown and G. M. Rebeiz, "A varactor-tuned RF filter", IEEE Trans Microwave Theory Tech., vol. 48, pp. 1157-1160, 2000.

[5] M. Makimoto and M. Sagawa, "Varactor tuned bandpass filters using microstrip-line ring resonators", IEEE Int. Symp. Microwave Theory Tech. Digest, pp. 411- 414, 1986.

[6] B. W. Kim and S. W. Yun, "Varactor-tuned combline bandpass filter using step-impedance microstrip lines", IEEE Trans. Microwave Theory Tech. 52, pp. 1279-1283, 2004.

[7] D. R. Smith, W. J. Padilla, D. C. Vier, S. C. Nemat-Nasser, and S. Schultz, "Composite medium with simultaneously negative permeability and permittivity", Phys. Rev. Lett., vol. 84, pp. 41844187, 2000.

[8] K. Aydin and E. Ozbay, "Capacitor-loaded split ring resonators as tunable metamaterial components", J. Appl. Phys., 2007.

[9] I. Gil, J. Bonache, J. Garcia-Garcia, and F. Martin, "Tunable metamaterial transmission lines based on varactor-loaded split-ring resonators", IEEE Trans. Microwave Theory Tech., vol. 54, 26652674, 2006.

[10] J. S. Hong and M. J. Lancaster, Microstrip filters for RF/microwave applications, New York: Wiley, 2001.

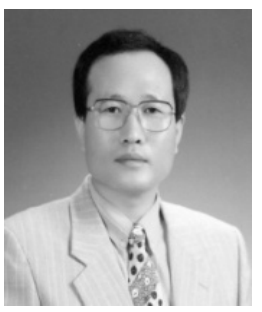

Girae Kim received the B.S., M.S. in Electronic Engineering from the Sogang University, Seoul, Korea, in 1986, 1988, respectively. He also received Ph.D. from Kyungnam University, Korea in 1999. From 1988 to 1993 he was a researcher in Communication Research Center of Samsung Electronics Co. Ltd. Since 1999, he has been on the faculty of Electronics Engineering Department at the Silla University, Busan, Korea. 